\title{
Análisis del Î́ndice Descriptivo del Trabajo (JDI) en trabajadores de instituciones de salud de Lima, Perú
}

\author{
Analysis of the job descriptive index in health care workers in Lima, Peru
}

\author{
Alicia Boluarte Carbajal ${ }^{1, a, b}$ \\ RESUMEN
}

Objetivos: Obtener indicadores de confiabilidad y de validez de constructo del Índice Descriptivo de Trabajo (JDI) mediante el método de análisis factorial. Material y métodos: Estudio de tipo psicométrico. El JDI está compuesto por 72 ítems agrupados en cinco dimensiones: Trabajo en general, Sueldo, Compañeros, Supervisión y Promoción. Además, se cuenta con una escala adicional que mide la satisfacción laboral de manera general. Esta escala se denomina Trabajo en General (JIG) y suele aplicarse junto con el JDI. La muestra estuvo conformada por 166 trabajadores de tres instituciones públicas de salud de Lima. Se realizaron correlaciones inter-ítem, análisis factorial confirmatorio en cada una de las dimensiones y consistencia interna con el coeficiente omega. Resultados: Se encontró que las dimensiones del JDI funcionan adecuadamente, aunque fue necesario eliminar algunos ítems y permitir errores correlacionados en algunas subescalas. En general, los ítems mostraron buena capacidad discriminativa y dispersión. Los estimadores de confiabilidad para las subescalas del JDI se hallaron entre 0,86 y 0,94 . Conclusiones: El JDI parece mostrar adecuadas propiedades estructurales y sus puntajes pueden usarse para propósitos descriptivos del contexto laboral. La escala complementaria JIG mostró un pobre funcionamiento psicométrico, lo cual indica que requiere reformulación, así como una reevaluación de su estructura factorial controlando el efecto del método (fraseo de los ítems).

PALABRAS CLAVE: Satisfacción en el trabajo, índices, análisis factorial, reproducibilidad de los resultados. (Fuente: DeCS BIREME).

\section{SUMMARY}

Objectives: To obtain reliable indicators of the job descriptive index (JDI) through a factorial analysis. Methods: A psychometric study was conducted. The JDI is composed of 72 items grouped in five dimensions: general work, salary, partners, supervision and promotion. In addition a general scale measures overall satisfaction. The samples size was 166 workers of three public health institutions in Lima. Factorial analysis and internal consistency analysis with the omega coefficient were carried-out. Results: The dimensions worked properly. The items showed good discriminatory capacity. Reliability indicators were 0.86 and 0.94 . Conclusions: JDI is adequate to evaluate conditions at work. The complementary scale need to be re-evaluated.

KEYWORDS: Job Satisfaction, indexes, factor analysis, statistical, reproducibility of results. (Source: MeSH BIREME).

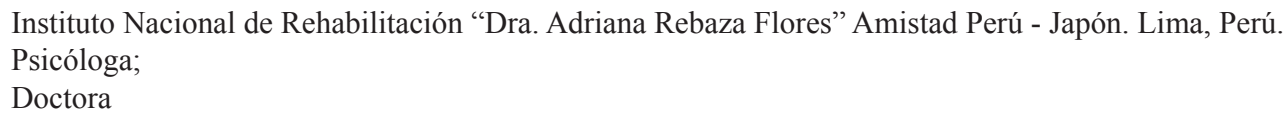




\section{INTRODUCCIÓN}

El estudio de la satisfacción laboral (SL) se enmarca dentro de una disciplina científica y multidisciplinar llamada comportamiento organizacional. Esta disciplina aporta al entendimiento de la dinámica y los procesos laborales en una organización. Asimismo, se orienta no solo a desarrollar un mejor trabajador, sino también una mejor persona, teniendo en cuenta que el trabajo modela a la persona y su beneficio trasciende de la empresa a la sociedad (1).

La satisfacción laboral es vista como el producto de las actitudes de los trabajadores hacia diferentes aspectos de su trabajo, como el salario, la supervisión, las condiciones de trabajo, las oportunidades de ascenso, entre otros (2). Se trata de una actitud afectiva favorable o desfavorable sobre su actividad laboral y resulta de la evaluación de las características de su puesto de trabajo $(1,3)$.

La medición del concepto se basa en dos métodos ampliamente utilizados: la medida global y la división por factores que supone la estructuración de escalas multidimensionales. La literatura científica demuestra que ambos métodos son útiles (3). Sin embargo, Rutherford et al (4), señalan que las escalas globales de satisfacción laboral no brindan una información precisa y completa de la satisfacción de los trabajadores, sino que proveen de una información mínima en aspectos específicos del ambiente laboral. Por el contrario, las escalas multidimensionales han reportado efectos diferenciales en cuanto a las actitudes y comportamientos de los trabajadores, proporcionando información más valiosa respecto a la satisfacción laboral. Si bien las propuestas globales ofrecen una medida rápida, el riesgo en la medición global es que los trabajadores suelen imitar comportamientos grupales, por lo que pueden no reflejar con objetividad las actitudes del trabajador. Por este motivo, el estudio de la SL se aprecia mejor desde una perspectiva multidimensional, en la cual es útil un análisis de cada uno de los elementos relacionados con el trabajo (1). Este enfoque brinda a los gerentes información sobre la causa de los problemas a fin de poder resolverlos con mayor rapidez y previsión (3).

En razón a ello, diversas investigaciones en el extranjero han trabajado con el Índice Descriptivo de Trabajo (JDI, siglas en inglés del Job Descriptive Index) con el fin de analizar la satisfacción laboral (5). Este instrumento es muy utilizado alrededor del mundo, especialmente en Estados Unidos. Por ejemplo, se ha usado para estudiar los niveles de satisfacción laboral en ambientes educativos, como escuelas primarias (6), y secundarias (7). También ha sido empleado en el sector de salud pública (8), así como en militares (9). Sin embargo, en Perú, no se reportan investigaciones sobre las propiedades métricas del JDI en alguna población. Es necesario desarrollar estudios que permitan validar instrumentos de medición orientados, especialmente, al estudio de la problemática organizacional de las instituciones de salud pública, pues dicha problemática repercute en la calidad de la atención a la población debido a las graves deficiencias institucionales existentes (10). Por los motivos expuestos, esta investigación tuvo como objetivo obtener indicadores de confiabilidad y de validez de constructo del Índice Descriptivo de Trabajo (JDI) mediante el método de análisis factorial (11).

\section{MATERIAL Y MÉTODOS}

Estudio transversal, prospectivo y de diseño psicométrico, entendida como una rama de la psicología orientada al análisis de las propiedades métricas exigibles de validez y confiabilidad que todo instrumento de medición debe tener (12).

La muestra total estuvo conformada por 166 trabajadores, entre asistenciales y administrativos, con más de 2 años de antigüedad, nombrados y contratados, quienes fueron seleccionados de manera no probabilística de tres instituciones de salud de Lima Metropolitana: el Instituto Nacional Rehabilitación Dra. Adriana Rebaza Flores (INR; $\mathrm{n}=88$ ), el Hospital Nacional Dos de Mayo (HNDM; $\mathrm{n}=61$ ) y el Instituto Nacional de Ciencias Neurológicas (INCN; $\mathrm{n}=17$ ). En la submuestra INR, el promedio de edad fue 44,32 $\pm 8,33$ y los participantes fueron predominantemente mujeres $(78,4 \%)$. En la submuestra HNDM, el promedio de edades fue $42,93 \pm 12,63$ y también se contó principalmente con mujeres $(80,3 \%)$ y en la submuestra INCN, el $82,35 \%$ fueron mujeres y la edad promedio de $41,81 \pm 7,51$.

Se utilizó el Índice Descriptivo de Trabajo (JDI), escala multidimensional que mide los siguientes aspectos de la SL: Trabajo en su Empleo Actual (TEA), Sueldo Actual (SA), Oportunidades de Ascenso (OA), Supervisión (S) y Compañeros de Trabajo (CT). Además, cuenta con una escala adicional que mide la SL de manera general. Esta escala se denomina Trabajo en General (JIG, por sus siglas en inglés) y suele aplicarse juntamente con el JDI. Las escalas 
se responden en un formato de tres respuestas ("Sín", "No" y "¿?"), las cuales pueden obtener alguno de los siguientes tres puntajes: 0,1 y 3 . Para el presente estudio, se utilizó la versión en español de Lacot, López y Martínez (13).

Se obtuvo autorización de los Comités de Ética y otras autoridades competentes de las instituciones de las que se extrajo la muestra. El procedimiento de recolección de datos se realizó mediante acercamientos a los departamentos, servicios y oficinas explicándoles previamente el propósito del estudio. A quienes aceptaron colaborar y participar voluntariamente se les entregó la hoja de consentimiento informado, se aseguró el anonimato y la confidencialidad de los datos recabados.

Se realizaron análisis psicométricos básicos (correlaciones ítem-test corregidas) en las submuestras INR y HNDM por separado. Se eliminaron los ítems que mostraron una pobre discriminación en ambas submuestras antes de proceder al análisis factorial con la muestra total (INR, HNDM, INCN).

A continuación, se realizó una serie de análisis factorial confirmatoria (AFC) con cada una de las subescalas del JDI y con la escala JIG. El estimador utilizado fue el de mínimos cuadrados diagonalmente ponderados basado en matrices policóricas y ajustado a la media y la varianza (WLSMV). Debido a que cada subescala fue planteada para evaluar un constructo específico (p. ej., la satisfacción con los compañeros de trabajo), el AFC de cada una de ellas partió de un modelo unidimensional. El ajuste se evaluó a través de diversos índices: CFI $>0,90$; TLI $>0,90$; RMSEA $<0,06$; WRMR < 0,95; sin embargo, es importante tomar en cuenta que estos valores son referenciales y la decisión final sobre el ajuste debe basarse en criterios conceptuales. En caso de que el ajuste fuera inadecuado, se procedió a revisar los índices de modificación (IM).

Se calculó, para cada solución factorial, el coeficiente omega como una medida de la confiabilidad (14). Cuando se observaron errores correlacionados se procedió a aplicar una corrección al cálculo del coeficiente Omega (15). En los casos en que las recomendaciones brindadas por los IM no contaron con sustento teórico suficiente o no resultaron suficientes para lograr un buen ajuste en el AFC, se procedió a realizar un análisis factorial exploratorio (AFE) con el estimador de mínimos cuadrados no ponderados basado en matrices policóricas. Finalmente, se analizaron las correlaciones entre los puntajes de las escalas del JDI y de la JIG.

Todos los análisis del presente estudio se realizaron en el programa $\mathrm{R}$ versión 3.3.3 (16) utilizando los paquetes Lavaan (17) y Psych (18).

\section{RESULTADOS}

Se realizó un AFC de la subescala TEA luego de eliminar los ítems 14 y 16, ya que estos obtuvieron correlaciones ítem-total bajas en las submuestras INR y HNDM. En el ajuste del AFC, se observaron niveles inadecuados: WLSMV $\chi^{2}(104)=253,12, p<0,001$, CFI $=0,84$, TLI $=0,82$, RMSEA $=0,09$, WRMR $=1,29$.

En los IM, se apreció la necesidad de permitir la correlación entre los errores de los ítems 2 ("Rutinario") y 17 ("Repetitivo"). Cuando se permitió dicha correlación $\left(\phi_{2-17}=0,79\right)$, el ajuste del modelo mejoró de manera notoria: WLSMV $\chi^{2}(103)=173.24$, $p<0,001$, CFI $=0,93$, TLI $=0,91, \quad$ RMSEA $=0,06$, WRMR $=1,00$.

El coeficiente omega corregido por la presencia de errores correlacionados, fue $\omega=0,88$. Sin embargo, se apreciaron cargas factoriales bajas en algunos ítems, especialmente en el caso del ítem 11 ("Cansador", $\lambda=$ 0,11 ); el promedio de cargas factoriales fue 0,58 .

En el AFC con la subescala SA luego de eliminar el ítem 5 debido a su baja correlación ítem-total en las submuestras INR y HNDM, los índices de ajuste indicaron que el modelo unidimensional se ajustó adecuadamente a los datos: $\operatorname{WLSMV} \chi^{2}(20)=32,68$, $p<0,001, \quad$ CFI $=0,97, \quad$ TLI $=0,96, \quad$ RMSEA $=0,06$, WRMR $=0,70$. El promedio de cargas factoriales fue 0,65 , siendo la más baja la correspondiente al ítem 6 ("Es inseguro", $\lambda=0,31$ ). La confiabilidad de la escala a partir de las cargas factoriales estandarizadas, alcanzó un valor $\omega=0,86$.

El análisis de la OA, mostró un ajuste aceptable: $\operatorname{WLSMV}^{2}(27)=63,86, p<0,001, \mathrm{CFI}=0,95$, $\mathrm{TLI}=0,93$, RMSEA $=0,09$, WRMR $=0,88$. Los IM sugirieron que se permitiera la correlación de los errores de los ítems 7 ("Los ascensos son poco frecuentes") y 8 ("Hay ascensos con regularidad"), lo cual tiene sentido conceptualmente debido a que ambos ítems hacen referencia a la frecuencia de los ascensos. Al permitir esta correlación $\left(\phi_{7-8}=0,46\right)$, se obtuvo un ajuste adecuado: $\operatorname{WLSMV} \chi^{2}(26)=41,94$, $p=0,03$, CFI $=0,98$, TLI $=0,97$, RMSEA $=0,06$, 
$\mathrm{WRMR}=0,70$. El promedio de cargas factoriales fue 0,66 y el coeficiente de confiabilidad corregido por espuriedad fue $\omega=0,86$.

En el AFC de la subescala $\mathrm{S}$, se encontró un ajuste aceptable: WLSMV $\chi^{2}(135)=278,06, p<0,001$, $\mathrm{CFI}=0,96$, TLI $=0,958$, RMSEA $=0,08$, WRMR $=$ 1,04. El coeficiente de confiabilidad estimado a partir de la solución factorial fue $\omega=0,95$. El promedio de cargas factoriales fue 0,71 . Sin embargo, se observó una carga factorial baja en el ítem 8 ("No supervisa suficientemente", $\lambda=0,28$ ).

Tabla 1. Características sociodemográficas.

\begin{tabular}{lccc}
\hline & INR & HNDM & INCN \\
& $\mathrm{N}(\%)$ & $\mathrm{N}(\%)$ & $\mathrm{N}(\%)$ \\
\hline Sexo & & & \\
Femenino & $69(78,4)$ & $49(80,3)$ & $14(82,35)$ \\
Masculino & $19(21,60)$ & $12(19,70)$ & $3(17,64)$ \\
Ocupación & & & \\
Asistencial & $72(81,81)$ & $43(70,49)$ & $13(76,47)$ \\
Administrativo & $16(18,18)$ & $18(29,51)$ & $4(23,52)$ \\
Total & 88 & 61 & 17 \\
\hline
\end{tabular}

INR: Instituto Nacional de Rehabilitación;

HNDM: Hospital Nacional Dos de Mayo

INCN: Instituto Nacional de Ciencias Neurológicas
Al modelar los ítems de la subescala CT en una única variable latente, se alcanzó un ajuste aceptable: $\operatorname{WLSMV}^{2}(135)=241,73, p<0,001, \mathrm{CFI}=0,96$, TLI $=0,95$, RMSEA $=0,07, \mathrm{WRMR}=1,02$. Las cargas factoriales se hallaron entre 0,46 (ítem 9) y 0,83 (ítem 11 ), y el promedio de ellas fue 0,69 . El coeficiente de confiabilidad obtenido fue igual a 0,94 .

El AFC unidimensional del JIG, mostró un ajuste inaceptable: WLSMV $\chi^{2}(135)=349,19, p<0,001$, $\mathrm{CFI}=0,82, \mathrm{TLI}=0,79, \mathrm{RMSEA}=0,10, \mathrm{WRMR}=1,56$. Asimismo, el examen de los IM no indicó la posibilidad de una modificación justificable conceptualmente. Por tal motivo, se procedió a realizar un AFE que permitiera vislumbrar la multidimensionalidad presente en los datos. Se utilizó el método de mínimos cuadrados no ponderados y el análisis se basó en correlaciones policóricas.

Para decidir la cantidad de factores a retener, se tomó como guía el criterio de Kelley (19). obteniendo un valor de $1 / 166^{1 / 2}=0,08$. Al analizar la solución de un factor, como se esperaba según los resultados del AFC, se halló un ajuste inadecuado ( $\mathrm{RMSR}=0,18)$. Por otra parte, tanto la solución de dos factores como la de tres brindaron valores de RMSR que se hallaron en torno al criterio de Kelley (0,09 y 0,06, respectivamente). En la tabla 1 , se presentan ambas soluciones factoriales.

Tabla 2. Análisis factorial exploratorio de la escala JIG.

\begin{tabular}{|c|c|c|c|c|c|c|c|}
\hline \multirow{2}{*}{ Ítem } & \multicolumn{3}{|c|}{ Solución de dos factores } & \multicolumn{4}{|c|}{ Solución de tres factores } \\
\hline & $\mathrm{F} 1$ & $\mathrm{~F} 2$ & $h^{2}$ & $\mathrm{~F} 1$ & $\mathrm{~F} 2$ & F3 & $h^{2}$ \\
\hline 1. Agradable & 0,43 & 0,37 & 0,42 & $-0,11$ & 0,06 & 0,87 & 0,72 \\
\hline 2. Malo & 0,61 & 0,10 & 0,42 & 0,14 & $-0,15$ & 0,74 & 0,60 \\
\hline 3. Ideal & $-0,11$ & 0,78 & 0,58 & $-0,27$ & 0,63 & 0,32 & 0,59 \\
\hline 4. Una pérdida de tiempo & 0,80 & 0,04 & 0,66 & 0,54 & $-0,03$ & 0,42 & 0,65 \\
\hline 5. Bueno & 0,69 & 0,24 & 0,62 & 0,30 & 0,06 & 0,63 & 0,68 \\
\hline 6. Indeseable & 0,90 & $-0,17$ & 0,75 & 0,81 & $-0,11$ & 0,16 & 0,77 \\
\hline 7. Vale la pena & 0,42 & 0,53 & 0,58 & 0,43 & 0,57 & 0,08 & 0,64 \\
\hline 8. Peor que la mayoría & 0,75 & $-0,31$ & 0,53 & 0,79 & $-0,18$ & $-0,04$ & 0,61 \\
\hline 9. Aceptable & 0,60 & 0,06 & 0,39 & 0,32 & $-0,05$ & 0,44 & 0,41 \\
\hline 10. Superior & $-0,11$ & 0,77 & 0,55 & 0,06 & 0,87 & $-0,16$ & 0,69 \\
\hline 11. Mejor que la mayoría & $-0,22$ & 0,61 & 0,35 & $-0,08$ & 0,67 & $-0,14$ & 0,40 \\
\hline 12. Desagradable & 0,66 & 0,39 & 0,74 & 0,37 & 0,27 & 0,50 & 0,75 \\
\hline 13. Estoy contento con mi trabajo & 0,38 & 0,65 & 0,70 & 0,17 & 0,52 & 0,41 & 0,69 \\
\hline 14. Inadecuado & 0,61 & 0,22 & 0,50 & 0,47 & 0,20 & 0,26 & 0,50 \\
\hline 15. Excelente & $-0,15$ & 0,77 & 0,54 & $-0,06$ & 0,78 & -v03 & 0,58 \\
\hline 16. Pésimo & 0,81 & $-0,04$ & 0,63 & 0,76 & 0,02 & 0,12 & 0,68 \\
\hline 17. Lo disfruto & 0,27 & 0,77 & 0,79 & 0,04 & 0,61 & 0,45 & 0,79 \\
\hline 18. Inferior & 0,72 & $-0,02$ & 0,51 & 0,89 & v15 & $-0,16$ & 0,74 \\
\hline
\end{tabular}


Tabla 3. Matriz de correlaciones de las subescalas del JDI y la escala JIG.

\begin{tabular}{lcccccc} 
& TEA & SA & OA & S & CT & JIG \\
\hline TEA & 1 & & & & & \\
\hline SA & 0,14 & 1 & & & & \\
OA & 0,28 & 0,31 & 1 & & & \\
S & 0,34 & 0,15 & 0,36 & 1 & & \\
CT & 0,24 & 0,05 & 0,15 & 0,28 & 1 & \\
JIG & 0,43 & 0,11 & 0,31 & 0,29 & 0,39 & 1 \\
\hline
\end{tabular}

Al analizar la solución de dos factores, se aprecia que todos los ítems de puntuación inversa $(2,4,6,8$, $14,16,18)$ cargaron en un solo factor. A excepción de los ítems 1, 5, 7 y 9, todos los ítems redactados de manera positiva cargaron en el segundo factor. La correlación entre ambos factores fue de 0,29.

En el caso de la solución de tres factores, el primer factor estuvo conformado por los ítems redactados de manera negativa. Por el contrario, el segundo factor comprendió, principalmente, ítems redactados de manera muy favorable para el trabajo de la persona (p. ej., "superior", "excelente"). Finalmente, el tercer factor presentó un contenido más variado, aunque en su mayoría estuvo compuesto por ítems de redacción más neutral que los anteriores. Las correlaciones inter factoriales fueron las siguientes: 0,14 (F1-F2), 0,44 (F1-F3) y, 34 (F2-F3).

Finalmente, se examinaron las correlaciones entre los puntajes del JDI y del JIG. Dichos puntajes se obtuvieron sumando los ítems correspondientes a cada dimensión. Como se observa en la tabla 2, se encontraron correlaciones bajas entre las subescalas. Esto indicó un buen poder discriminativo de estas, debido a que las correlaciones bajas demuestran que cada dimensión es relativamente independiente. Sin embargo, en contra de lo esperado, la correlación entre la subescala TEA y la escala JIG fue solo de nivel moderado.

\section{DISCUSIÓN}

Se examinó la validez de constructo de la escala JDI y su complemento, la escala JIG, en una muestra de profesionales procedentes de instituciones de salud de Lima. Los análisis se realizaron por cada dimensión, debido a que cada una fue planteada para evaluar un constructo específico. La bibliografía señala que la satisfacción laboral en el trabajador depende de varios factores $\mathrm{y}$, por lo tanto, puede apreciarse mejor desde un enfoque multidimensional y no como una puntuación general (1).
Se realizó el AFC de las escalas del JDI con la finalidad de demostrar la uni dimensionalidad de cada uno de los factores, a fin de poner a prueba que cada uno de los ítems medía un mismo constructo, lo cual ha sido demostrado por otros autores $(9,13)$. Los resultados del presente estudio indican que los ítems del JDI pueden agruparse en sus escalas hipotetizadas con buenos niveles de poder discriminativo. En un estudio realizado con trabajadores chinos y trabajadores estadounidenses, se demostró que cada dimensión del JDI poseía una estructura unidimensional, lo que se evidencia también en la presente investigación (20).

Los resultados indican que el instrumento parece ser, en esta etapa de su evaluación métrica, una herramienta satisfactoria para la descripción e investigación de la satisfacción laboral en centros de salud pública, pues los niveles de confiabilidad generalmente han sido buenos y sugieren que el monto de error de medición puede ser tolerable para propósitos descriptivos grupales, Teniendo en cuenta que el coeficiente alfa se ve influenciado por el número de ítems (21), el número de alternativas de respuesta y la proporción de la varianza del test, fue pertinente utilizar el coeficiente omega recomendado como un estimador de la confiabilidad alternativa al coeficiente alfa (14). Los índices de confiabilidad reportados fueron aceptables y se encontró similitud con otros estudios $(13,22)$, los cuales hallaron una fluctuación del alfa de 0,79 a 0,87 . También en un metaanálisis realizado con 152 estudios para analizar la validez del constructo del JDI, se encontraron adecuadas consistencias internas en las dimensiones, con los siguientes valores promedio del coeficiente de confiabilidad: $\mathrm{TEA}=0,88, \mathrm{SA}=0,87, \mathrm{OA}=0,88, \mathrm{~S}=$ 0,89 y $\mathrm{CT}=0,86$ (23).

En la escala JIG emergieron entre dos y tres subgrupos, lo que pareciera significar que su interpretación debería considerar más de una dimensión. Esto no fue coherente con el diseño de construcción de la escala JIG. Una explicación para 
este problema podría ser el fraseo negativo de alguno de sus ítems, pues uno de los factores de estas escalas retuvo ítems fraseados de esta manera. Es usual que estos problemas emerjan cuando un instrumento contiene ítems con sus contenidos formulados en dirección positiva (hacia el concepto medido) y negativa (opuesto al concepto medido) $(24,25)$.

Otra explicación podría deducirse del análisis de correlación (tabla 3) realizado entre cada dimensión, en el cual se halló que la correlación entre la escala TEA y la JIG fue de un nivel moderado $(r=0,43)$ en comparación con las otras dimensiones que mostraron ser bajas. Este resultado concuerda con el estudio de McIntyre et al, quienes encontraron correlaciones entre bajas a moderadas, a excepción de la escala de TEA, la cual mostró una correlación alta $(0,70)$ con la escala JIG (22). También en otro estudio se encontró una correlación alta entre estas escalas: 0,72 (26). Estos resultados concuerdan con lo observado en el presente estudio, lo que da cuenta de que cada factor es independiente uno de otro y que la escala JIG puede ser redundante respecto de la escala TEA, asumiendo que los participantes podrían haber observado dicha similitud o también haberse sentido sobrecargados (27) por la cantidad de ítems a resolver, hecho común cuando se trata de escalas multidimensionales en entornos organizacionales.

Las limitaciones del estudio se refieren a varios aspectos. Primero, el tamaño muestral debe ser más grande para asegurar de la estabilidad de los resultados; segundo, no se puede tener garantía de la representatividad de la muestra debido que no se hizo algún procedimiento aleatorio en su selección, por tal motivo se maximizó la participación de los sujetos. Otra de las limitaciones a considerar fue el formato de respuesta, en el que se incluyó la escala JIG, la cual es complementaria al JDI, pues otros estudios la aplican en forma separada. También que la población de estudio fue predominantemente de sexo femenino.

Este instrumento es por primera vez utilizado en Perú mediante el procedimiento del AFC. Se ha demostrado que el modelo multidimensional para la evaluación de la SL, utilizado por la escala JDI, es adecuado y puede afirmarse que las características evaluadas son satisfactorias para dirigir otros estudios que verifiquen sus correlatos con criterios como la intención de dejar el trabajo, estrés laboral, entre otros. Finalmente, la escala JIG demostró que tiene una estructura factorial inestable, poco clara, por lo que no se recomienda el uso por el momento y resulta necesario realizar otros estudios que muestren la replicabilidad de los resultados del JDI.

\section{Fuentes de financiamiento y declaración de conflictos de interés:}

El estudio fue financiado por el Instituto Nacional de Rehabilitación "Dra. Adriana Rebaza Flores" Amistad Perú-Japón. La autora declara que no hay conflictos de intereses de algún tipo.

\section{Correspondencia:}

Alicia Boluarte Carbajal.

Jr. Julio Rodavero 890 Pueblo Libre, Lima, Perú

Correo electrónico: aliciaboluarte@gmail.com

Celular: 51- 984088660

\section{REFERENCIAS BIBLIOGRÁFICAS}

1. Newstrom JW. Comportamiento humano en el trabajo. Ciudad de México: McGraw-Hill; 2011. p.573.

2. Blum ML, Naylor JC. Psicología Industrial: sus fundamentos teóricos y sociales. Ciudad de México: Trillas; 1999.

3. Robbins SP, Judge TA. Comportamiento organizacional. Ciudad de México: Pearson; 2013. p.712.

4. Rutherford B, Boles J, Hamwi GA, Madupalli R, Rutherford $\mathrm{L}$. The role of the seven dimensions of job satisfaction in salesperson's attitudes and behaviors. J Bus Res. 2009; 62(11):1146-51.

5. Castanheira F. Job Descriptive Index. En: Michalos A, editor. Encyclopedia of Quality of Life and WellBeing Research. Dordrecht: Springer Netherlands; 2014. p. 3439-41.

6. Paswaters R. A study of Florida Public Elementary School Principals' Job satisfaction following the implementation of Florida's A+ System for Grading Schools. Florida: University of Central Florida; 2006.

7. Lanzo A. An assessment of the level of job satisfaction of middle school teachers from selected affluent suburban public-school districts in Essex County. Thesis. New Jersey: Seton Hall University; 2003.

8. Luddy N. Job satisfaction amongst employees at a public health institution in the Western Cape. Tesis. Ciudad del Cabo: University of the Western Cape; 2005. (Citado el 15 de junio del 2018) Disponible en: http://etd.uwc.ac.za/xmlui/handle/11394/1418

9. Lopes S, Chambel MJ, Castanheira F, Oliveira-Cruz F. Measuring job satisfaction in Portuguese military sergeants and officers: Validation of the job descriptive index and the job in general scale. Mil Psychol. 2015; 27(1):52-63. 
10. Frenk J, Chen L, Bhutta ZA, et al. Profesionales de la salud para el nuevo siglo: transformando la educación para fortalecer los sistemas de salud en un mundo interdependiente. Rev Peru Med Exp Salud Publica. 2011; 28(2):337-41.

11. Kerlinger FN, Lee HB. Investigación del comportamiento. Ciudad de México: McGraw-Hill; 2006. p.808.

12. Aragón L. Fundamentos Psicométricos en la Evaluación Psicológica. Rev Electrónica Psicol Iztacala. 2004;7(4): 23-43.

13. Lacot I, Lopez N, Martinez-Lugo M. El índice descriptivo del empleo: traducción, adaptación $\mathrm{y}$ validación en una muestra de empleados en Puerto Rico. Rev Puertorriquena Psicol. 1991;7(1):93-9.

14. Ventura-león JL, Caycho-Rodríguez T. El coeficiente Omega: un método alternativo para la estimación de la confiabilidad. Rev Latinoam Ciencias Soc Niñez y Juv. 2017; 15(1):625-7.

15. Domínguez-Lara SA. Errores correlacionados y estimación de la fiabilidad en estudios de validación: comentarios al trabajo de validación de la escala Ehealth Literacy (EHEALS) en población universitaria española. Rev Esp Salud Publica. 2016; 90: e1-e2. (Citado el 15 de junio del 2018) Disponible en: http://scielo.isciii.es/scielo.php?script=sci_arttext \&pid $=$ S1135-57272016000100602

16. R Core Team. R: A language and environment for statistical computing. Viena: $\mathrm{R}$ Foundation for Statistical Computing; 2017.

17. Rosseel Y. lavaan: An R Package for Structural Equation Modeling. J Stat Softw. 2012; 48(2):1-36.

18. Revelle W. psych: Procedures for Personality and Psychological Research. Evanston: Northwestern University; 2017.

19. Ferrando PJ, Anguiano-Carrasco C. El análisis factorial como técnica de investigación en psicología. Papeles del Psicólogo. 2010;31(1):18-33.
20. Wang M, Russell SS. Measurement equivalence of the job descriptive index across Chinese and American workers: Results from confirmatory factor analysis and item response theory. Educ Psychol Meas. 2005;65(4):709-32.

21. Nunnally JC, Bernstein IJ. Teoría psicométrica. Ciudad de Mexico: McGraw-Hill; 1995.

22. McIntyre SE, McIntyre TM. Measuring job satisfaction in portuguese health professionals: Correlates and validation of the job descriptive index and the job in general scale. Int J Selecetion Assess. 2010;18(4):425-31.

23. Kinicki A, Mckee-Ryan F, Schriesheim C, Carson K. Assessing the construct validity of the Job Descriptive Index: A review and meta-analysis. J Appl Psychol. 2002; 87(1): 14-32

24. Schmitt N, Stuits DM. Factors defined by negatively keyed items: The result of careless respondents? Appl Psychol Meas. 1985;9(4):367-73.

25. Spector PE, Van Katwyk PT, Brannick MT, Chen PY. When two factors don't reflect two constructs: how item characteristics can produce artifactual factors. J Manage. 1997;23(5):659-77.

26. Russell SS, Spitzmüller C, Lin LF, Stanton JM, Smith PC, Ironson GH. Shorter can also be better: the abridged job in general scale. Educ Psychol Meas. 2004; 64(5): 878-93.

27. Rogelberg SG, Conway JM, Sederburg ME, Spitzmüller C, Aziz S, Knight WE. Profiling active and passive nonrespondents to an organizational survey. J Appl Psychol. 2003;88(6):1104-14.

Recibido: 02/07/2018

Aceptado: 27/12/2018 